Nephrologe 2010 [Suppl 1] 5:3-4 DOI 10.1007/s11560-010-0407-4

(c) Springer-Verlag 2010

\section{D.E. Müller-Wiefel}

Pädiatrische Nephrologie und $\mathrm{KfH}-$ Kindernierenzentrum, UKE, Universitätsklinikum Hamburg-Eppendorf

\title{
Die Kindernephrologie ist erwachsen geworden
}

Die pädiatrische Nephrologie hat sich bei uns, aber auch international in ein Stadium entwickelt, wo sie die von ihr geforderte Eigenständigkeit lebt und klinisch wie wissenschaftlich tagtäglich mit hoher Qualifikation unter Beweis stellt.

Mit der Fixierung einer institutionalisierten 3-jährigen Ausbildung eines Pädiaters zum Kindernephrologen in der Musterweiterbildungsordnung der Bundesärztekammer 2003 in Form eines Schwerpunktes bzw. einer Zusatzweiterbildung (je nach Bundesland) hat sich die Kindernephrologie auch gesundheitspolitisch voll etabliert. Der Ausbildungskatalog zum Kindernephrologen stellt heute auch die Basis für den kassenärztlichen Versorgungsauftrag nierenkranker Kinder dar. Der Weiterbildungsinhalt umfasst im Einzelnen den Erwerb von Kenntnissen, Erfahrungen und Fertigkeiten in

- der Erkennung und Behandlung angeborener wie erworbener, einschließlich glomerulärer und tubulärer Funktionsstörungen und Erkrankungen von Niere und Harntrakt,

- der Erkennung und Behandlung akuter und chronischer Nierenfunktionsstörungen,

- der Erkennung und Behandlung der arteriellen renalen Hypertonie sowie der renalen Osteopathie und Anämie,

- den hormonellen Veränderungen einschließlich Wachstumsstörungen bei Kindern und Jugendlichen mit Nierenerkrankungen,

- der interdisziplinären Indikationsstellung für urologisch-chirurgische Behandlungsverfahren,
- der Vorbereitung, prä- und postoperativen Versorgung von Kindern mit Nierentransplantation sowie deren Langzeitbetreuung,

- Doppler-/Duplex-Untersuchungen der Nierengefäße, auch nach Transplantation,

- der Nierenbiopsie,

- extrakorporalen Blutreinigungsverfahren bei Intoxikationen, Stoffwechselerkrankungen und Stoffwechselkrisen,

- der Peritonealdialyse sowie

- der Hämodialyse und verwandten Techniken, wie Filtration, Adsorption und Separation.

Dabei sind Richtzahlen für die Sonographie, die verschiedenen Dialyseformen, die Transplantationsbetreuung in den verschiedenen Behandlungsstadien und die Nierenbiopsien vorgegeben.

Mittlerweile sind mehr als 90 nach diesen Richtlinien von den Landesärztekammern akkreditierten Kindernephrologen in die Versorgung nierenkranker Kinder integriert, in der Regel in Kliniken, da die Beteiligung in freien Praxen aufgrund der beschränkten Patientenzahlen in Relation zum hohen personellen und technologischen Aufwand ökonomisch irrelevant erscheint. Diese Aussage gilt insbesondere für die Kinderdialyse, für die seitens der Gesellschaft für Pädiatrische Nephrologie (GPN) umfassende Voraussetzungen erarbeitet wurden bezüglich Ausstattung (sämtliche Blutreinigungsverfahren, Intensivstation, Schulunterricht), Personal (Kindernephrologen, Kinderdialyseschwestern, psychosoziales Begleitteam) und Behandlungsfrequenz (HD, PD, TPL). So wird Kindernephrologie heute vorwiegend an Universitätskliniken praktiziert, was den Vorteil mit sich bringt, dass diese gleichzeitig als Ausbildungszentren fungieren können, sofern die von der Deutschen Akademie für Kinder- und Jugendmedizin erarbeiteten spezifischen Strukturanforderungen für Weiterbildungsstätten erfüllt sind. Diese Ausbildungszentren bilden in aller Regel auch die Zellen der wissenschaftlichen Aktivitäten, die sich immer nur am kranken Patienten orientieren können. Es bleibt zu hoffen, dass die Ökonomisierungsbestrebungen - leider auch auf dem kindernephrologischen Sektor - diese intakte, fruchtbare Funktionalität aus Krankenversorgung, Weiterbildung und Forschung in der universitären Kindernephrologie nicht zerstören, wenngleich erste Ansätze dazu sich unschwer erkennen lassen.

Die 41. Jahrestagung der Gesellschaft für Pädiatrische Nephrologie (GPN) vom 25.-27.03.2010 in Hamburg soll Anlass zur Präsentation eines Sonderheftes „Pädiatrische Nephrologie“ von „Der Nephrologe" sein. Mit diesem bietet die Kindernephrologie Einblick in ihre aktuellen Aktivitäten und zwar zum einen in Form von Übersichtsarbeiten zu spezifisch kindernephrologischen Themen, zum anderen durch die zur Vortrags- bzw. Posterpräsentation auf dem Kongress ausgewählten Abstracts. Dabei stehen nicht ausschließlich die Hauptthemen Niere und Hormone, kongenitale Nephro- und Uropathien sowie Nierenersatztherapie im Fokus, sondern auch die beiden pädiatriecharakteristischen Krankheitsbilder 
hämolytisch-urämisches und nephrotisches Syndrom. Die Übersichten orientieren sich an den Kongresshauptthemen: Einleitend wird in einem bisher einzigartigen Beitrag die Geschichte der Entwicklung und Reifung der Kindernephrologie in der Bundesrepublik geschildert. Dann wird der Weg zur immer bedeutenderen Subdisziplin der Neonatalnephrologie beschritten, die mit der Genetik samt ihrer tagespolitischen Relevanz durch die aktuelle Gesetzgebung unweigerlich verknüpft ist. Es folgt eine Abhandlung zur pädiatriespezifischen Problematik der Wachstumshormontherapie bei Niereninsuffizienz, bevor ein Musterbeispiel an weltweiter globaler Kinderperitonealdialysetherapie anhand eines internationalen Registers gegeben wird, mit dem abschließend eine höchst kritische Auseinandersetzung mit der Vergabe von Spendernieren für pädiatrische Empfänger verknüpft wird.

Möge Ihnen die Lektüre der ausgesuchten Manuskripte mit einem Blick in die Abstraktrubrik den Einblick in die Kindernephrologie lebendig und überzeugend gestalten - auch überzeugend dafür, dass die Kindernephrologie in ihrem Wirkungsfeld zwar erwachsen geworden ist, sich aber durchaus als integrativen Bestandteil der nephrologischen Gesamtheit in unserem Lande betrachtet.

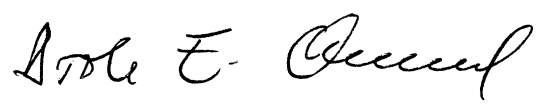

\section{Prof. Dr. D. E. Müller-Wiefel}

Vorsitzender der Gesellschaft für Pädiatrische Nephrologie (GPN) und Tagungspräsident der 41. wissenschaftlichen Jahrestagung der GPN

\section{Korrespondenzadresse Prof. Dr. D.E. Müller-Wiefel}

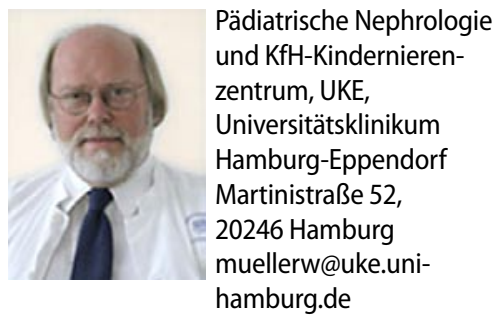

\section{Themenübersicht}

„Der Nephrologe“ bietet Ihnen umfassende und aktuelle Beiträge zu interessanten Themenschwerpunkten aus allen Bereichen der Nephrologie und Hypertensiologie.

\begin{tabular}{|c|c|c|}
\hline \multirow{2}{*}{ Der Nephrologe } & \multicolumn{2}{|c|}{ Leitthemen 2009} \\
\hline & - Heft 1/09 & Bildgebende Diagnostik in der Nephrologie \\
\hline$=$ & - Heft 2/09 & Akutes Nierenversagen \\
\hline & - Heft 3/09 & Langzeitbetreuung nach Transplantation \\
\hline & - Heft 4/09 & Schwangerschaft und Niere \\
\hline & $=$ Heft 5/09 & $\begin{array}{l}\text { Knochen- und Mineralstoffwechselstörungen } \\
\text { bei Nierenkranken }\end{array}$ \\
\hline & - Heft 6/09 & Niere und Hypertoni \\
\hline & Leitthemen 2 & 10 \\
\hline Der & $=$ Heft $1 / 10$ & Grenzbereiche der Nephrologie \\
\hline$\frac{3}{6 x}(2)$ & $\begin{array}{l}- \text { Heft } 2 / 10 \\
- \text { Heft } 3 / 10 \\
- \text { Heft } 4 / 10 \\
- \text { Heft } 5 / 10 \\
- \text { Heft } 6 / 10\end{array}$ & $\begin{array}{l}\text { Transplantation und Immunsuppression } \\
\text { Dialyse } \\
\text { Chronische interstitielle Nierenerkrankungen } \\
\text { Zystische Nierenerkrankungen } \\
\text { Nierenbiopsie }\end{array}$ \\
\hline
\end{tabular}

\section{Volltextarchiv „Der Nephrologe“}

Abonnenten haben online Zugriff auf alle Beiträge im elektronischen Volltextarchiv unter www.DerNephrologe.de

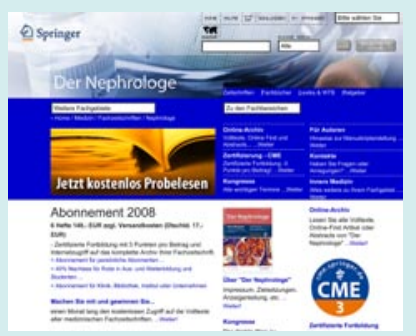

Ins Volltextarchiv gelangen Sie von der Startseite über den Navigationspunkt "Online-Archiv“. Nach einer einmaligen Registrierung können Sie die Beiträge als pdf oder in der html-Version abrufen.

Haben Sie noch Fragen oder Anmerkungen? Wir freuen uns über Ihre Email an: birgit.wasser@springer.com

Ihre Redaktion

Fachzeitschriften Medizin/Psychologie 